\title{
Hepatitis $B$ virus $x$ protein induces epithelial-mesenchymal transition of hepatocellular carcinoma cells by regulating long non-coding RNA
}

Yinji $\mathrm{Jin}^{1+}$, Di Wu ${ }^{2+}$, Weiwei Yang ${ }^{1}$, Mingjiao Weng ${ }^{1}$, Yafei $\mathrm{Li}^{1}$, Xuefei Wang ${ }^{1}$, Xiao Zhang ${ }^{1}$, Xiaoming Jin ${ }^{1 *}$ and Tianzhen Wang ${ }^{1 *}$

\begin{abstract}
Background: It has been widely accepted that hepatitis B virus $X$ protein $(\mathrm{HBx})$ plays an important role in hepatocellular carcinoma (HCC). This study aimed to explore the function of long non-coding RNAs (IncRNAs) in the epithelial-mesenchymal transition (EMT) induced by HBx.

Methods: The association between HBx and EMT markers was detected using immunohistochemistry in HCC tissues. The effect of HBx on HCC EMT was assessed through morphological analysis, transwell assay, metastatic in vivo study and detection of EMT markers. LncRNA microarray was used to screen the differently expressed IncRNAs. Small interfering RNA and Western blot were used to analyse the function and mechanism of the locked IncRNA.

Results: HBx was negatively correlated with the epithelial marker E-cadherin but positively correlated with the mesenchymal marker vimentin in HCC tissues. HBx induced the mesenchymal phenotype and improved the metastatic ability of HCC cells. Meanwhile, HBx down-regulated E-cadherin, whereas it up-regulated vimentin. In HCC cells, HBX altered the expression of 2002 IncRNAs by more than 2-fold. One of them was ZEB2-AS1. Inhibition of ZEB2-AS1 can compensate for the EMT phenotype and reverse the expression of EMT markers regulated by HBx. Additionally, HBX affected the Wnt signalling pathway.
\end{abstract}

Conclusions: HBx promotes HCC cell metastasis by inducing EMT, which is at least partly mediated by IncRNAs.

Keywords: Hepatocellular carcinoma, Hepatitis B virus x protein, Long non-coding RNA, Epithelial-mesenchymal transition, Metastasis

\section{Background}

Hepatocellular carcinoma (HCC) is one of the most common cancers with high mortality worldwide [1]. Hepatitis B virus (HBV) infection is regarded as the leading risk factor for the development of HCC [2]. The rate of metastasis and recurrence of HCC in patients with HBV infection is significantly higher than patients without $\mathrm{HBV}$ infection [3-5]. Most studies attribute the oncogenic function of HBV to the HBV x protein $(\mathrm{HBx})$, which is encoded by HBV $\mathrm{X}$ gene and has been proven

\footnotetext{
*Correspondence: Jinxm55@163.com; wtzpath@163.com

${ }^{\dagger}$ Equal contributors

'Department of Pathology, Harbin Medical University, Harbin 150081, China

Full list of author information is available at the end of the article
}

to promote $\mathrm{HCC}$ cells metastasis by regulating matrix metalloproteinases (MMPs), cell adhesion molecules, microRNAs (miRNAs), and so on [6,7].

Epithelial-mesenchymal transition (EMT) is an important mechanism for cancer metastasis and recurrence [8]. The cancer cells undergoing EMT will acquire mesenchymal cell-like features and show some changes in morphology, molecular markers and metastatic ability [9]. Lee et al. found that $\mathrm{HBx}$ mediated the activation of STAT5b, which further improves cancer cell motility and invasiveness by inducing EMT in HCC [10]. Yang et al. reported that $\mathrm{HBx}$ induced EMT by activating c-Src in HCC cells [11]. Until now, some molecules have been 
proven to be involved in the regulation of EMT; however, the mechanism is still not well known.

In recent years, increasing evidence indicates that long non-coding RNAs (lncRNAs) play an important role in cancer development [12]. HBx has been identified to promote $\mathrm{HCC}$ proliferation and metastasis by regulating lncRNA expression [13, 14]. HULC is the first lncRNA with highly specific up-regulation in HCC [15]. Liu et al. found that single nucleotide polymorphisms (SNPs) in HULC decreased the susceptibility to HCC in HBV persistent carriers [16]. Anin vitro study indicated that HBx up-regulated HULC expression and then promoted HCC proliferation by suppressing p18 [17]. Subsequently, some lncRNAs were found to play a role in HCC development, including Dreh, LINE, and HOTAIR [18-20].

In this study, we determined the association between HBx and EMT in HCC tissues. Then, we detected the effect of HBx on the metastatic ability and EMT markers of HCC cells. Moreover, lncRNAs, which are regulated by $\mathrm{HBx}$ and mediate the oncogenic function of $\mathrm{HBx}$, were determined in HCC cells. It is important for understand the mechanism of HCC metastasis and explore novel targets for treatment.

\section{Methods}

\section{Tissue microarray}

The tissue microarray was obtained from the Superchip Biotechnology Company in Shanghai. The microarray contains $47 \mathrm{HCC}$ samples. None of the patients received any therapy prior to surgery, and all the patient samples had complete clinical information.

\section{Immunohistochemistry}

Immunohistochemistry was performed on the tissue microarray slide conventionally. In brief, the slide was deparaffinized by xylene and rehydrated using a graded ethanol series. Then, $3 \% \mathrm{H}_{2} \mathrm{O}_{2}$ was used to block endogenous peroxidase in the tissues. Antigen retrieval was completed using microwave treatment. Then, 5\% bovine serum albumin was used to block nonspecific reactions. The slides were incubated with primary antibody against E-cadherin (SCBT, Santa Cruz, CA, USA, 1:100), vimentin (SCBT, 1:100) and HBx (US Biological, Swampscott, MA, USA, 1:100) at $4{ }^{\circ} \mathrm{C}$ overnight. The streptavidin-biotin peroxidase kit (ZSGB Bio, Beijing, China) was used to detect the bound antibodies. Finally, the band was visualized by DAB staining (ZSGB Bio).

The immunohistochemical result was scored using the intensity and extent. Staining intensity was quantified as follows: negative (0), weak (1), moderate (2) or strong (3). Staining extent was quantified according to the percentage of positive cells: none (0), <25\% (1), $25-50 \%$ (2),
$50-75 \%$ (3) or $>75 \%$ (4). The final score was calculated as the intensity score multiplied by the extent score.

\section{Cell culture}

The HCC cell line HepG2 and lentiviral vector packaging cell line 293TN were grown in Dulbecco's modified Eagle medium (DMEM; HyClone, Logan, UT, USA) with 10\% fetal bovine serum (FBS; Invitrogen, Carlsbad, CA, USA), $100 \mu \mathrm{g} / \mathrm{ml}$ streptomycin and $100 \mathrm{IU} / \mathrm{ml}$ penicillin at $37^{\circ} \mathrm{C}$ in a humidified atmosphere containing $5 \% \mathrm{CO} 2$.

\section{Immunocytochemistry}

Cells were seeded in 24 well plates, where the slides were placed in advance. After $24 \mathrm{~h}$, the slides were fixed with $10 \%$ formalin and permeabilized with $0.1 \%$ Triton $\mathrm{X}-100$. Then, the slides were incubated with $\mathrm{HBx}, \mathrm{E}-$ cadherin and vimentin antibodies at $37{ }^{\circ} \mathrm{C}$ for $2 \mathrm{~h}$. The secondary antibodies with streptavidin-biotin peroxidase were used to detect the primary antibodies. Finally, the binding was visualized by DAB staining.

\section{Generation of stable cell line}

The HBx overexpression lentiviral vector was constructed by inserting the synthetic $\mathrm{HBx}$ (genotype C) into CON238 vectors. The pseudo lentivirus was packaged according to a previous report [21]. To generate stable cells, HepG2 cells were incubated with $10 \mathrm{MOI}$ of control lentiviral particles or HBx overexpression lentiviral particles for at least $12 \mathrm{~h}$, then the GFP positive cells were sorted with a flow cytometry after $72 \mathrm{~h}$ of culture in normal medium.

\section{Transfection}

Cells were seeded in 6 well plates, and the small interfering RNAs (siRNAs) (Invitrogen) of the LncRNAs were transfected into cells using Lipofectamine 2000 (Invitrogen) according to the manufacturer's protocol. The scrambled sequence was used as a negative control (NC). The siRNA-ZEB2-AS1 sequence used was 5'-CAAAGGACACCTTTGGTT ACCTGAA-3' [22].

\section{Transwell assays}

The cell migration and invasion assays were performed using transwell chambers with or without Matrigel (Becton Dickson, Bedford, MA, USA). Briefly, $2 \times 10^{4}$ cells (HepG2-control and HepG2-HBx) suspended in serum-free media were plated in the upper chamber. DMEM media with 10\% FBS was plated in the bottom chamber. The chamber was incubated at $37^{\circ} \mathrm{C}$ for $24 \mathrm{~h}$, and then the bottom of the chamber was stained with crystal violet. Cells were counted in five randomly selected 200x fields under light microscopy. The average cell number was used to reflect the invasive and migratory ability. 


\section{Xenograft metastatic in vivo study}

Twenty healthy BALB/c female nude mice (4-6 weeks old) were purchased from Beijing Vital River Laboratory Technology Co., Ltd. (China), and the experiments were conducted according to the applicable national and international guidelines. They were randomly divided into two groups receiving HepG2-control and HepG2HBx cells, respectively. Then, $1 \times 10^{6}$ cells were suspended in $100 \mu \mathrm{L}$ of PBS and injected into the nude mouse via the tail vein. Eight weeks later, lung tissue was collected for detection of the number and volume of the metastatic tumours by pathological methods.

\section{Western blot}

Protein was extracted from the cultured cells and was quantitated using a BCA assay (Beyotime, Haimen, China). Equal amounts of protein were separated by SDS-PAGE and transferred onto PVDF membranes. The membranes were incubated with anti-E-cadherin (1:500), anti-vimentin (1:500), anti-HBx (1:200), anti-ZEB2 (1:200) and anti-GAPDH (SCBT, 1:2000) at $4{ }^{\circ} \mathrm{C}$ overnight, and then they were incubated with the respective secondary antibody at $37{ }^{\circ} \mathrm{C}$ for $2 \mathrm{~h}$. Resulting bands were detected by an ECL Western blotting detection system (Thermo Scientific, Rockford, IL, USA).

\section{RNA extraction and LncRNAs microarray}

Total RNA was isolated from the cultured cells (HepG2control and HepG2-HBx) using TRIzol reagent (Invitrogen). The A260/A280 was detected by a Nano Drop ND2000 spectrophotometer and RNA bands separated by agarose gel electrophoresis were used to determine the quantity and quality of the RNA. A260/A280 $\geq 1.9$ and clearly visible bands were confirmed in all the samples before further experiments. Microarray hybridization was performed by Shanghai Biotechnology Corporation using a Sure Print G3 human lncRNA $4 \times 180$ k V6 microarray (Agilent).

\section{Real time-PCR validation assay}

The level of lncRNA was validated by real time PCR using a SYBR green PCR mix (TransGen Biotech, Beijing, China) and BIO-RAD CFX96 ${ }^{\text {Tn }}$ Real-Time System. LncRNA levels were normalized to GAPHD. PCR conditions were as follows: denaturation at $95{ }^{\circ} \mathrm{C}$ for $5 \mathrm{~min}, 39$ cycles of amplification at $95^{\circ} \mathrm{C}$ for $10 \mathrm{~s} / \mathrm{cycle}$, and then $60{ }^{\circ} \mathrm{C}$ for $30 \mathrm{~s}$. Melting curve analyses were performed on the PCR products by progressive heating from $65{ }^{\circ} \mathrm{C}$ to $95{ }^{\circ} \mathrm{C}$. Primers (GenePharma Co., Shanghai, China) specific for the lncRNAs and GAPDH were as follows: ZEB2-AS1, forward (F) 5'-ATGAAGA AGCCGCGAAGTGT-3' and reverse (R) 5' -CACACCCTAATACACATGCCCT-3', and GAPDH, forward (F)
5'-ATGGGGAAGGTGAAGGTCG-3' and reverse(R) 5'-GGGGTCATTGATGGCAACAATA-3' [22, 23].

\section{Statistical analysis}

All the data were presented as the mean \pm SD. Student'stest was used to compare the difference between the two groups. The relationship between the two groups was analysed by the Pearson correlation test. $P<0.05$ was considered to indicate a statistically significant difference. The DAVID Bioinformatics Tool (https://david.ncifcrf.gov/, version 6.7) was used to complete the functional enrichment analysis of the gene ontology (GO) and Kyoto encyclopaedia of genes and genomes (KEGG) database. GO functional terms were limited to "Biological Process" and KEGG pathways with FDR < 0.05 were considered to be significant.

\section{Results}

$\mathrm{HBx}$ was associated with EMT in HCC

To determine the association between HBx and EMT in $\mathrm{HCC}$, we detected $\mathrm{HBx}$ and EMT markers in the HCC tissues by immunohistochemistry. Of $47 \mathrm{HCC}$ samples, the level of E-cadherin was lower in the HCC tissues with $\mathrm{HBx}$ (33 cases) than those without HBx (14 cases), whereas the level of vimentin was on the opposite side (Table 1). HBx was negatively correlated with E-cadherin but positively correlated with vimentin (Fig. 1). The correlation coefficient was -0.65 between $\mathrm{HBx}$ and $\mathrm{E}$ cadherin, while it was 0.70 between $\mathrm{HBx}$ and vimentin. This result indicated that HBx had an effect on EMT in HCC.

\section{HBx can promote HCC cell migration and invasion by regulating EMT}

To identify the effect of HBx on EMT in HCC, we constructed an HBx-overexpressed HepG2 cell line by lentivirus infection and detected the cellular morphology, metastatic ability and expression of EMT markers. Under light microscopy, we found that $\mathrm{HBx}$ induced morphological change from the epithelial to mesenchymal phenotype (Fig. 2a). Transwell assays showed that $\mathrm{HBx}$ promoted the migratory and invasive ability of HepG2 cells. The number of cells passed through the wells was significantly higher in the HBx overexpressed HepG2 cells than the control cells (Fig. 2b-e). In vivo study showed that metastatic tumours formed in $70 \%$ (7/10) of the nude mice injected with the HepG2control but in 100\% (10/10) of those with HepG2-HBx. The number and size of the metastatic tumours also predominated in the HepG2-HBx group (Fig. 2f).The immunocytochemical result indicated that the epithelial marker of E-cadherin was down-regulated, whereas the mesenchymal marker of vimentin was up-regulated in the HBx overexpressed HepG2 cells (Fig. 2g). These data 
Table 1 The association between HBx and E-cadherin, vimentin and clinical parameters in HCC

\begin{tabular}{llll}
\hline Parameters & $\begin{array}{l}\text { HBx negative } \\
(n=14)\end{array}$ & $\begin{array}{l}\text { HBx positive } \\
(n=33)\end{array}$ & $P$ Value \\
\hline Age & $50.0 \pm 8.7$ & $49.7 \pm 9.3$ & \\
Sex (male/female) & $13 / 1$ & $27 / 6$ & \\
Grade & & & \\
$\quad$ I-II & 8 & 21 & \\
$\quad$ III-IV & 6 & 12 & \\
Tumour size (diameter) & & & \\
$\quad 3$ & & \\
$>3$ cm & 7 & 14 & \\
AFP ( $\geq 300$ ng/L) & 6 & 11 & \\
Creatinine $(\mu \mathrm{mol} / \mathrm{L})$ & $68.0 \pm 16.5$ & $72.7 \pm 40.5$ & \\
Urea nitrogen $(\mathrm{mmol} / \mathrm{L})$ & $5.3 \pm 0.6$ & $5.2 \pm 1.4$ & $<0.05$ \\
CEA (ng/ml) & $3.9 \pm 2.3$ & $3.1 \pm 1.6$ & $<0.05$ \\
E-cadherin & $6.5 \pm 2.5$ & $4.1 \pm 1.8$ & \\
Vimentin & $3.5 \pm 2.1$ & $6.2 \pm 3.1$ & \\
\hline
\end{tabular}

indicated that $\mathrm{HBx}$ might promote the metastatic ability of HCC cells by inducing EMT.

\section{HBx might regulate EMT by affecting the expression of IncRNAs in HCC}

To explore the potential function of lncRNAs in $\mathrm{HBx}-$ regulated EMT, we compared the different expression of the lncRNAs between the HepG2-control and HepG2HBx. The result indicated that 2002 LncRNAs were down-regulated or up-regulated by more than 2-fold in the HepG2 cells because of HBx. Of them, the ZEB2AS1 (ZEB2-antisense RNA1), a known-EMT related lncRNA, was up-regulated by 3.55 -fold. We first verified the function of ZEB2-AS1 in HepG2 cells. Knockdown of ZEB2-AS1 inhibited the metastatic ability of HepG2 cells (Fig. 3a and b). We further verified the level of ZEB2-AS1 by Q-PCR and that it was indeed increased in HepG2-HBx (Fig. 3c). Moreover, the metastatic ability was decreased in HepG2-HBx when ZEB2-AS1 was down-regulated by the siRNA technique (Fig. $3 \mathrm{~d}$ and e). Western blot assays indicated that $\mathrm{HBx}$ up-regulated vimentin and down-regulated E-cadherin; however, knockdown of ZEB2-AS1 in HepG2-HBx reversed their expression (Fig. 3f). More interestingly, ZEB2, as one of the key regulatorsthat mediated the function of ZEB2-AS1, was also decreased with knockdown of ZEB2-AS1(Fig. 3f). That is, knockdown of ZEB2-AS1 can compromise the EMT process induced by $\mathrm{HBx}$. Taken together, $\mathrm{HBx}$ might promote the EMT process by up-regulating the expression of ZEB2-AS1.

\section{HBx affected EMT associated genes expression in HCC}

Additionally, the microarray results also showed the different expression of mRNAs between the HepG2control and HepG2-HBx. Two hundred sixty-two mRNAs were down-regulated and 197 mRNAs were up-regulated by HBx in the HepG2 cells. GO enrichment analysis revealed that all the altered mRNAs were significantly enriched in $41 \mathrm{GO}$ terms (Fig. 4a),

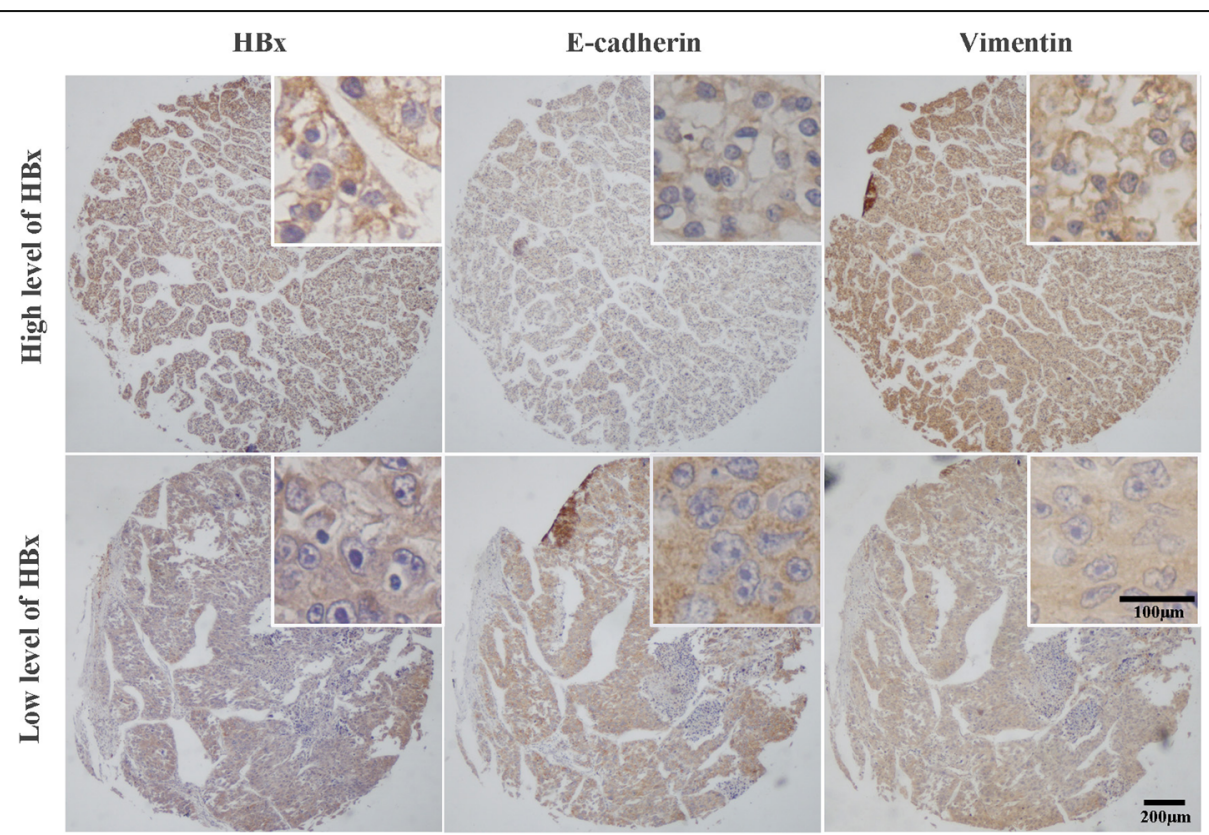

Fig. 1 The expression of HBx and EMT markers was detected by immuno- histochemistry on the HCC tissue microarray. The expression of E-cadherin was decreased but that of vimentin was increased in the HCC tissues with high levels of HBx compared to those with low levels of $\mathrm{HBx}(100 \times)$.The top right corner is the enlarged image (400x) 


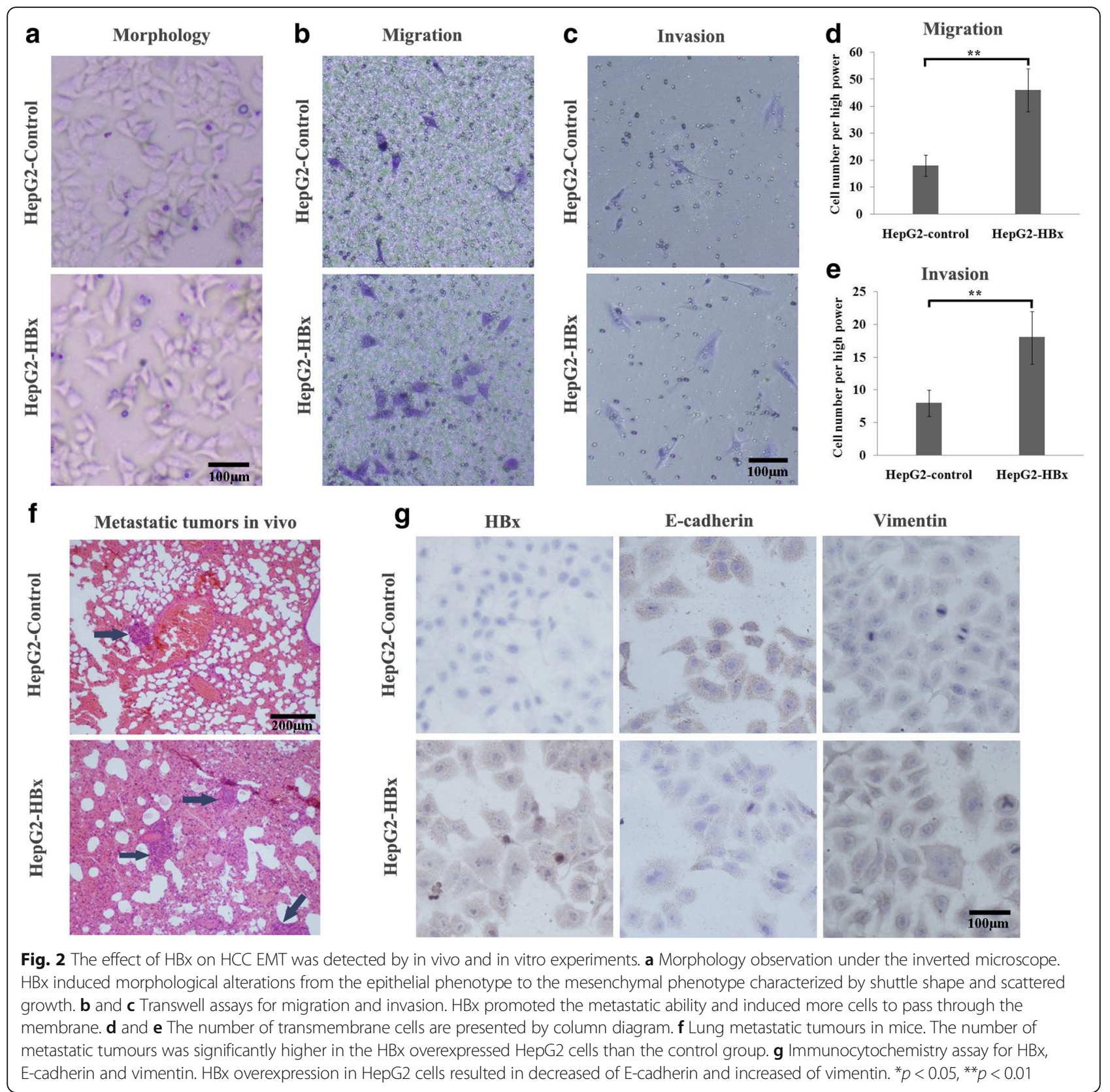

including cytoskeleton organization, and positive regulation of focal adhesion assembly. The KEGG pathway enrichment analysis indicated that five KEGG pathways were significantly enriched, in which the Wnt signalling pathway has been widely confirmed to play an important role in EMT (Fig. 4b). These results indicated that $\mathrm{HBx}$ regulated EMT associated genes expression.

\section{Discussion}

HBx plays an important role during the development of $\mathrm{HBV}$-associated HCC. Here, we verified that $\mathrm{HBx}$ can induce EMT in HCC. In 2006, Lee et al. first proved the effect of HBx on EMT. Their study found that HBx activated STAT5b, which further promoted cancer cell motility and invasiveness by inducing EMT [10]. Successively, the activation of c-Src, STAT3, Wnt, Akt and Notch1 were identified to mediate EMT induced by HBx [11, 24-27]. The importance of the Wnt signalling pathway was also presented by our result. In addition to the coding RNAs, $\mathrm{HBx}$ can also regulate lncRNA expression. Microarrays performed in mice and normal liver cells uncovered lots of IncRNAs altered by HBx $[20,28]$. Recently, IncRNAs were found to 


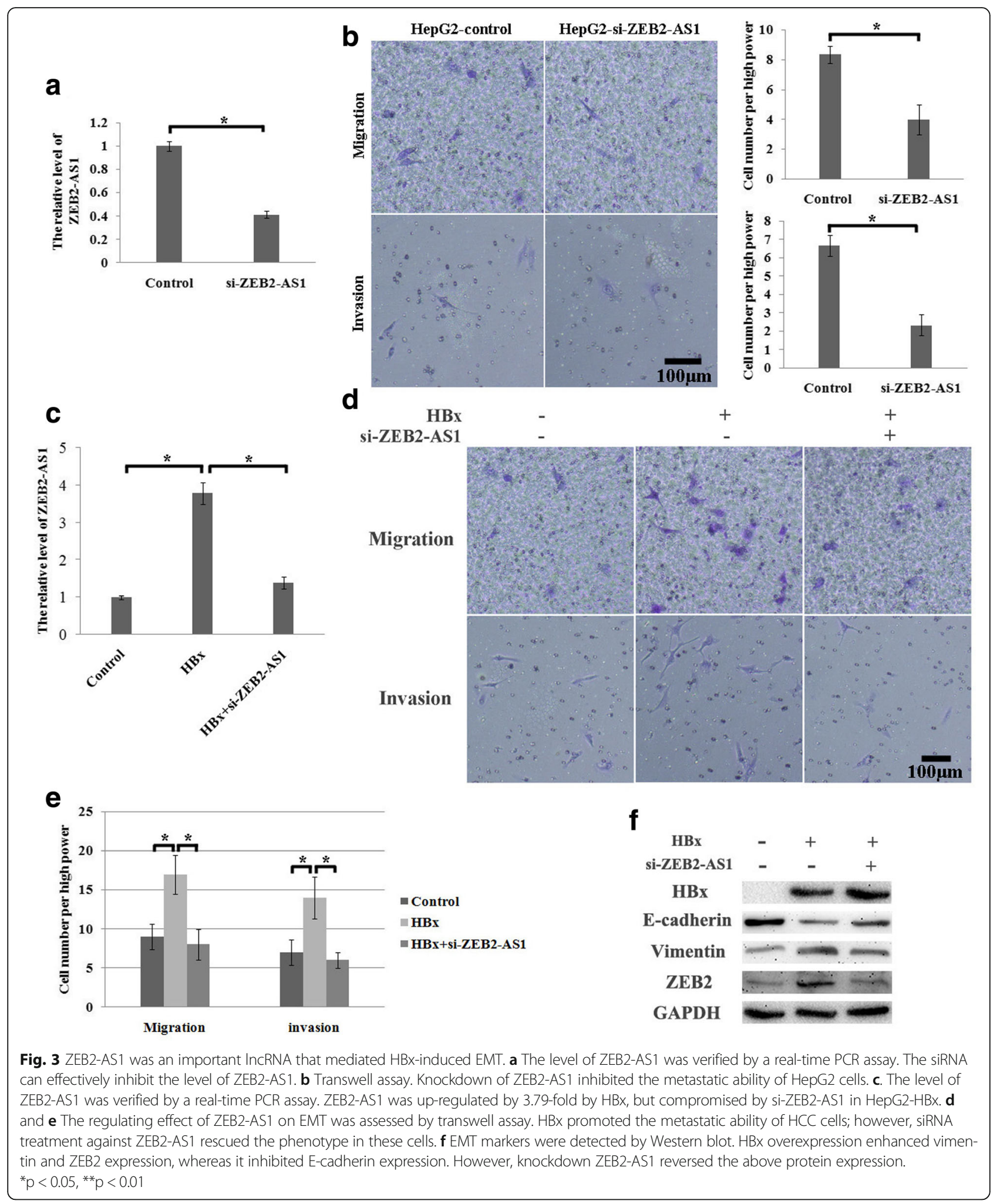

mediate HBx-induced EMT. HBX-LINE1 is a hybrid RNA transcript of the human LINE1 and the HBVencoded X gene, which promotes EMT-like changes by binding miR-122 [29]. Deng et al. found that HBx promoted EMT by enhancing the expression of Linc00152 in HCC [30]. Though we have these findings, we only know a little about the association between HBx and lncRNAs. 


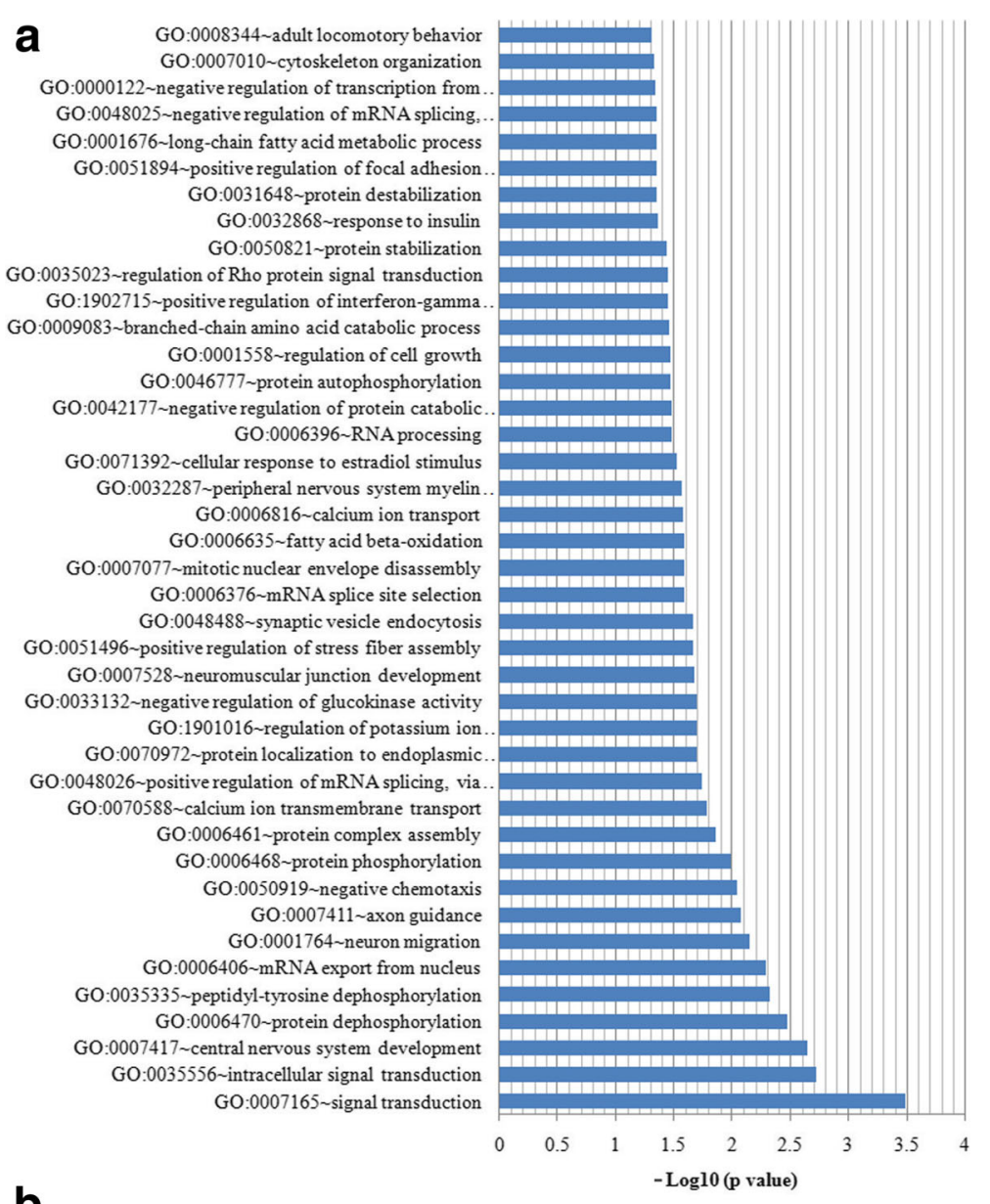

b

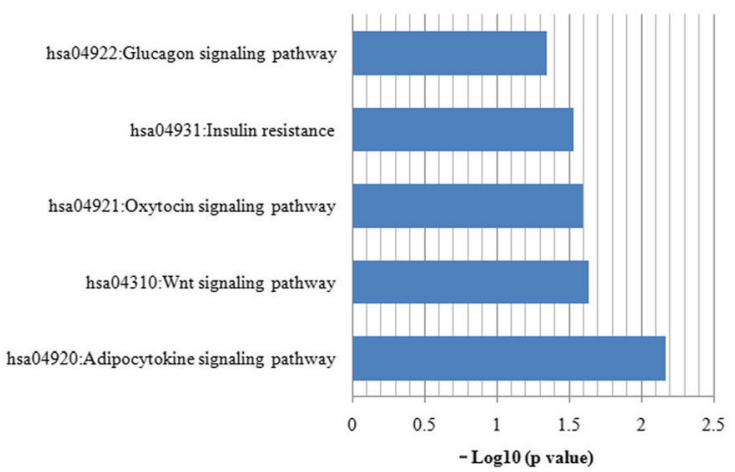

Fig. 4 Analysis for microarray results. a GO enrichment analysis. All the altered mRNAs were significantly enriched in $41 \mathrm{GO}$ terms. b KEGG pathway enrichment analysis. Five KEGG pathways were significantly enriched

To analyse the function of lncRNAs in EMT induced by $\mathrm{HBx}$, we compared the different expression of lncRNAs between HepG2-HBx and HepG2-control. The result indicated that multiple lncRNAs were altered. Of them, we have focused on ZEB2-AS1 in view of its association with EMT. ZEB2-AS1 is the natural antisense transcript corresponding to the 5'UTR of the zinc finger E-box binding homeobox 2. Over-expression of ZEB2AS1 in epithelial cells can prevent the splicing of the
Zeb2 $5^{\prime}$-UTR and up-regulate the levels of ZEB2 protein (a transcriptional repressor of E-cadherin) [31]. Consequently, E-cadherin was decreased and resulted in EMT [31]. It was found that TGF $\beta 1$ from the cancer associated fibroblasts can induce EMT in the urinary bladder cancer cells by up-regulating ZEB2-AS1 and ZEB2 protein level [32]. ZEB2-AS1was also increased in the HCC tissues, and high levels of ZEB2-AS1 were biomarkers for poor prognosis [22]. Further study indicated that 
ZEB2-AS1 promoted HCC growth and metastasis by regulating ZEB2 and some EMT markers [22]. In this study, we proved that $\mathrm{HBx}$ induced EMT and upregulated ZEB2-AS1 expression, whereas knockdown of ZEB2-AS1 compromised EMT in HBx overexpressed HepG2 cells. Therefore, ZEB2-AS1 mediated the occurrence of EMT induced by $\mathrm{HBx}$ in $\mathrm{HCC}$ cells. In addition to ZEB2-AS1, lots of lncRNAs were altered by $\mathrm{HBx}$ in HepG2 cells. These findings indicated that lncRNAs are involved in the development of HBV-associated HCC, though the functions of most of them are still not clear.

In this study, KEGG analysis divided the differentially expressed genes into five signalling pathways. Of them, the Wnt pathway is a well-known pathway associated with EMT, and the components of this pathway are involved in the EMT process [33, 34]. For example, $\beta$ catenin can translocate from the cytoplasm to the nucleus, where it regulates the expression of some genes associated with migration and invasion [35]. The activation of Wnt signalling can also up-regulate EMTassociated regulators, such as Snail and ZEB1 [36, 37]. Interestingly, it was reported that $\mathrm{HBx}$ can activate the Wnt signalling pathway by down-regulating the expression of the SFRPs (the antagonists of Wnt signalling pathway), thus promoting HBx-induced EMT [25]. Our result also indicated that HBX might promote EMT by activating the Wnt signalling pathway.

\section{Conclusions}

In conclusion, this study verified that $\mathrm{HBx}$ can promote HCC development by inducing EMT. LncRNAs involved in HBx-induced EMT and ZEB2-AS1 were identified as key lncRNAs mediating this process. $\mathrm{HBx}$ might upregulate ZEB2-AS1 and ZEB2, consequently inducing EMT. The Wnt signalling pathway was also associated with the occurrence of EMT induced by HBx. This study provided the evidence for the HBx-LncRNA-EMT axis. However, we do not have evidence with which to correlate ZEB2-AS1 and Wnt or other signalling pathways. In the complex network, there must be a certain link between the lncRNAs and all kinds of signalling pathways, which will be the goal of our explorations in the future.

\section{Abbreviations \\ DMEM: Dulbecco's modified Eagle medium; EMT: Epithelial-mesenchymal transition; FBS: Fetal bovine serum; GO: Gene ontology; HBV: Hepatitis B virus; HBx: HBV x protein; HCC: Hepatocellular carcinoma; KEGG: Kyoto encyclopaedia of genes and genomes; IncRNAs: Iong non-coding RNAs; MMPs: Matrix metalloproteinases; NC: Negative control; SNPs: Single nucleotide polymorphisms; ZEB2: Zinc finger E-box binding homeobox 2; ZEB2-AS1: ZEB2-antisense RNA1}

\section{Acknowledgements}

Not applicable.

\section{Funding}

This work was supported by the National Natural Science Foundation of China (Grant No. 81302061 to Tianzhen Wang), Postdoctoral Scientific
Research Development Fund of Heilongjiang Province (Grant No. LBH-Q15082 to Tianzhen Wang), Heilongjiang Postdoctoral Fund (Grant No: LBH-Z16118 to Di Wu), Heilongjiang Provincial Health Bureau (Grant No. 2014-411 to Yinji Jin) and the Fundamental Research Funds for the Provincial Universities (Grant No. 2017JCZX43 to Weiwei Yang).

\section{Availability of data and materials}

Not applicable.

\section{Authors' contributions}

Tianzhen Wang and Xiaoming Jin designed the study. Yinji Jin, Di Wu and Weiwei Yang performed the experiment. Mingjiao Weng and Feiya Li wrote the article. Xuefei Wang and Xiao Zhang analysed the data. All authors read and approved the final manuscript.

\section{Ethics approval and consent to participate}

All patients gave their informed consent prior to their inclusion in the study. This study was approved by the ethics committee of Harbin Medical University (reference numbers: HMUIRB20130089). This study was carried out in accordance with the National Institutes of Health guide for the care and use of Laboratory animals (NIH Publications No. 8023, revised 1978).

\section{Consent for publication}

Not applicable.

\section{Competing interests}

The authors declare that they have no competing interests.

\section{Publisher's Note}

Springer Nature remains neutral with regard to jurisdictional claims in published maps and institutional affiliations.

\section{Author details}

${ }^{1}$ Department of Pathology, Harbin Medical University, Harbin 150081, China. ${ }^{2}$ Department of Obstetrics and Gynecology, First Affiliated Hospital of Harbin Medical University, Harbin 150001, China.

Received: 28 August 2017 Accepted: 27 November 2017

Published online: 19 December 2017

\section{References}

1. Torre LA, et al. Global cancer statistics, 2012. CA Cancer J Clin. 2015; 65(2):87-108.

2. Zamor PJ, deLemos AS, Russo MW. Viral hepatitis and hepatocellular carcinoma: etiology and management. J GastrointestOncol. 2017;8(2):229-42.

3. Nanashima A, et al. Clinicopathological characteristics of patients with hepatocellular carcinoma after hepatectomy: relationship with status of viral hepatitis. J SurgOncol. 2007;96(6):487-92.

4. Yokoi Y, et al. Clinicopathological features of hepatocellular carcinomas (HCCs) arising in patients without chronic viral infection or alcohol abuse: a retrospective study of patients undergoing hepatic resection. J Gastroenterol. 2005:40(3):274-82.

5. Tanaka K, et al. Clinical characteristics and surgical outcome in hepatocellular carcinoma without hepatitis B virus surface antigen or hepatitis C virus antibody. Ann SurgOncol. 2007;14(3):1170-81.

6. Arzumanyan $\mathrm{A}$, et al. Epigenetic repression of E-cadherin expression by hepatitis B virus $x$ antigen in liver cancer. Oncogene. 2012;31(5):563-72.

7. Ou DP, et al. The hepatitis $B$ virus X protein promotes hepatocellular carcinoma metastasis by upregulation of matrix metalloproteinases. Int J Cancer. 2007;120(6):1208-14.

8. De Craene B, Berx G. Regulatory networks defining EMT during cancer initiation and progression. Nat Rev Cancer. 2013;13(2):97-110.

9. Iwatsuki M, et al. Epithelial-mesenchymal transition in cancer development and its clinical significance. Cancer Sci. 2010;101(2):293-9.

10. Lee TK, et al. Signal transducers and activators of transcription $5 b$ activation enhances hepatocellular carcinoma aggressiveness through induction of epithelial-mesenchymal transition. Cancer Res. 2006;66(20):9948-56.

11. Yang SZ, et al. HBx protein induces EMT through C-Src activation in SMMC7721 hepatoma cell line. BiochemBiophys Res Commun. 2009;382(3):555-60.

12. Weng $\mathrm{M}$, et al. Noncoding RNAs in the development, diagnosis, and prognosis of colorectal cancer. Transl Res. 2017;181:108-20. 
13. Yu FJ, et al. Long non-coding RNAs and hepatocellular carcinoma. Mol ClinOncol. 2015;3(1):13-7.

14. Moyo B, Nicholson SA, Arbuthnot PB. The role of long non-coding RNAs in hepatitis B virus-related hepatocellular carcinoma. Virus Res. 2016;212:103-13.

15. Panzitt K, et al. Characterization of HULC, a novel gene with striking upregulation in hepatocellular carcinoma, as noncoding RNA. Gastroenterology. 2007;132(1):330-42.

16. Liu Y, et al. A genetic variant in long non-coding RNA HULC contributes to risk of HBV-related hepatocellular carcinoma in a Chinese population. PLoS One. 2012;7(4):e35145.

17. Du Y, et al. Elevation of highly up-regulated in liver cancer (HULC) by hepatitis $B$ virus $X$ protein promotes hepatoma cell proliferation via downregulating p18. J Biol Chem. 2012;287(31):26302-11.

18. Zhang $\mathrm{H}$, et al. PLK1 and HOTAIR accelerate proteasomal degradation of SUZ12 and ZNF198 during hepatitis B virus-induced liver carcinogenesis. Cancer Res. 2015;75(11):2363-74.

19. Heikenwalder M, Protzer U. LINE(1)s of evidence in HBV-driven liver cancer. Cell Host Microbe. 2014;15(3):249-50.

20. Huang JF, et al. Hepatitis $B$ virus $X$ protein (HBx)-related long noncoding RNA (IncRNA) down-regulated expression by HBx (Dreh) inhibits hepatocellular carcinoma metastasis by targeting the intermediate filament protein vimentin. Hepatology. 2013;57(5):1882-92.

21. Zhi Q, et al. Oncogenic miR-544 is an important molecular target in gastric cancer. Anti Cancer Agents Med Chem. 2012;

22. Lan T, et al. Downregulation of ZEB2-AS1 decreased tumor growth and metastasis in hepatocellular carcinoma. Mol Med Rep. 2016;14(5):4606-12.

23. Wang $T$, et al. Lin28A enhances chemosensitivity of colon cancer cells to 5 -FU by promoting apoptosis in a let-7 independent manner. Tumour Biol. 2016;37(6):7657-65.

24. Teng J, et al. HBX-dependent activation of twist mediates STAT3 control of epithelium-mesenchymal transition of liver cells. J Cell Biochem. 2013;114(5):1097-104.

25. Xie Q, et al. Epigenetic silencing of SFRP1 and SFRP5 by hepatitis B virus X protein enhances hepatoma cell tumorigenicity through Wnt signaling pathway. Int J Cancer. 2014;135(3):635-46.

26. Ha HL, et al. IGF-II induced by hepatitis $B$ virus $X$ protein regulates EMT via SUMO mediated loss of E-cadherin in mice. Oncotarget. 2016;7(35):56944-57.

27. Wang $X$, et al. The deposition of Notch1 in hepatitis B virus-associated nephropathy and its role in hepatitis $B$ virus $X$ protein-induced epithelialmesenchymal transdifferentiation and immunity disorder in renal tubular epithelial cells. J Viral Hepat. 2014;21(10):734-43.

28. Kuang XY, et al. Expression profiles of long non-coding RNAs in human liver cell line LO2 with stable expression of hepatitis B x gene. Zhonghua Gan Zang Bing ZaZhi. 2016;24(6):417-21.

29. Liang HW, et al. Hepatitis B virus-human chimeric transcript HBX-LINE1 promotes hepatic injury via sequestering cellular microRNA-122. J Hepatol. 2016;64(2):278-91.

30. Deng $X$, et al. Linc00152 promotes cancer progression in hepatitis B virusassociated hepatocellular carcinoma. Biomed Pharmacother. 2017;90:100-8.

31. Beltran $\mathrm{M}$, et al. A natural antisense transcript regulates Zeb2/Sip1 gene expression during Snail1-induced epithelial-mesenchymal transition. Genes Dev. 2008;22(6):756-69.

32. Zhuang J, et al. TGFbeta1 secreted by cancer-associated fibroblasts induces epithelial-mesenchymal transition of bladder cancer cells through IncRNAZEB2NAT. Sci Rep. 2015;5:11924.

33. Liang Z, et al. Curcumin reversed chronic tobacco smoke exposure induced urocystic EMT and acquisition of cancer stem cells properties via Wnt/betacatenin. Cell Death Dis. 2017;8(10):e3066.

34. Wu C, et al. Interaction between Wnt/beta-catenin pathway and microRNAs regulates epithelial-mesenchymal transition in gastric cancer (review). Int J Oncol. 2016:48(6):2236-46.

35. Jia S, et al. KIAA1199 promotes migration and invasion by Wnt/beta-catenin pathway and MMPs mediated EMT progression and serves as a poor prognosis marker in gastric cancer. PLoS One. 2017;12(4):e0175058.

36. Yang Y, et al. Downregulated connexin32 promotes EMT through the Wnt/ beta-catenin pathway by targeting Snail expression in hepatocellular carcinoma. Int J Oncol. 2017, 1977-1988;50(6)

37. Bao $Z$, et al. CBX7 negatively regulates migration and invasion in glioma via Wnt/beta-catenin pathway inactivation. Oncotarget. 2017;

\section{Submit your next manuscript to BioMed Central and we will help you at every step:}

- We accept pre-submission inquiries

- Our selector tool helps you to find the most relevant journal

- We provide round the clock customer support

- Convenient online submission

- Thorough peer review

- Inclusion in PubMed and all major indexing services

- Maximum visibility for your research

Submit your manuscript at www.biomedcentral.com/submit

) Biomed Central 